\title{
Relaxor type perovskites: Primary candidates of nano-polar regions $^{\text {II }}$
}

\author{
S B KRUPANIDHI \\ Materials Research Centre, Indian Institute of Science, \\ Bangalore 560 012, India \\ e-mail: sbk@mrc.iisc.ernet.in
}

\begin{abstract}
Relaxor properties of $0 \cdot 7 \mathrm{~Pb}\left(\mathrm{Mg}_{1 / 3} \mathrm{Nb}_{2 / 3}\right) \mathrm{O}_{3}-0 \cdot 3 \mathrm{PbTiO}_{3}(\mathrm{PMN}-\mathrm{PT})$ and non-lead perovskite thin films have been analysed in terms of large frequency dispersion of dielectric response at low temperatures. A wide spectrum of dielectric relaxation was observed in the frequency-dependent response of the imaginary part of the dielectric permittivity. Transformation from normal ferroelectric to relaxor behaviour has been observed in the case of the Ca substituting the $\mathrm{BaTiO}_{3}$ thin films. A number of techniques were exploited to investigate the wide spectrum of relaxation times in pulsed laser ablated thin films. $a c$ and $d c$ electric field induced complex dielectric properties of $0.7 \mathrm{~Pb}\left(\mathrm{Mg}_{1 / 3} \mathrm{Nb}_{2 / 3}\right) \mathrm{O}_{3}-0.3 \mathrm{PbTiO}_{3}$ (PMN-PT) thin films were studied as function of frequencies at different temperatures. Nonlinear behaviour of dielectric susceptibility with respect to the amplitude of the $a c$ drive was observed at lower temperatures. The frequency dependence of transition temperature $T_{m}$ (temperature of the maximum of dielectric constant) was studied using the VogelFulcher relation.
\end{abstract}

Keywords. Relaxor; PMN-PT; BCT; PLD; Vogel-Fulcher.

\section{Introduction}

The potential of functional materials such as relaxor ferroelectrics has driven the research in the directions of integration into microsystems, medical instrumentation and microelectronic components. This is a result of the fast development in the fields of communication, microengineering and biomedical engineering. While having clear practical implications, however, advances in understanding of the basic properties of relaxor ferroelectrics present at the same time a fundamental scientific challenge.

The relaxor ferroelectrics are characterized by their diffuse nature of dielectric phase transition along with frequency dependent transition temperature. These materials also exhibit very high dielectric constant and electrostrictive coefficient and thus become one of the potential candidates for multilayer capacitors, micromechanical devices respectively. Despite the incessant endeavours for last one decade, the phenomenon of dielectric relaxation in relaxor materials is still meagrely understood. Different models have been proposed by the various authors to understand the diffuse phase transition of the relaxor materials. ${ }^{1-5}$ The evidence of relaxor nature in lead magnesium niobate was first observed by Smolenskii ${ }^{2}$ and the diffuse nature of phase transition was attributed to the randomly oriented polar microregions originated from compositional fluctuations on the nanometre length scale. These chemical fluctuations resulted in the statistical

\footnotetext{
"Dedicated to Professor C N R Rao on his 70th birthday
} 
distribution of the phase transition temperature and consequently the broad temperature dependence of dielectric properties. L E Cross ${ }^{1}$ further extended the Smolenskii's model to a superparaelectric model. An ideal superparaelectric could be considered as an ensemble of nano-dimensioned polar regions, the size of that are characterised by the spatial coherence of their spontaneous polarisation. Each nanopolar region was considered as the source of dipolar entity with no interaction among each other. The relaxor behaviour was attributed to these nanometer size regions to a thermally activated ensemble of superparaelectric clusters. The broad distribution of relaxation times for cluster orientations originates from the distribution of the potential barriers separating the different orientational states. The dielectric anomaly and frequency dispersion are due to slowing down of superparaelectric moments. A short-range cooperative interaction between these superparaelectric clusters was considered by Viehland et $a l^{6}$ to explain the freezing of the superparaelectric moments into a dipolar glassy with ferroelectric order at lower temperatures. Qian and Burshill $^{7}$ assumed that the nanometer scale chemical defects are the source of random fields, which control the dynamics of the polar clusters in such systems. They explained the broad dispersive behaviour by considering changes in the cluster size and correlation length as function of temperature. Structural origin of relaxor ferroelectric was discussed by Chen et $a l^{8}$ in terms of the chemical bonding between $\mathrm{Pb}$ and $\mathrm{O}$ ions. Kleeman et $a l^{5}$ however proposed that the charge compositional fluctuation quench the random electric fields, which were considered as the origin of diffuse phase transition in PMN relaxor and are also responsible for the critical slowing down of the dielectric relaxation. The freezing of dielectric relaxation in relaxor ferroelectric was directly evidenced by Gazounov and Tagantsev ${ }^{9}$ following the VogelFulcher law. In the present case both dielectric and ferroelectric properties of perovskitetype relaxor thin films have been studied to investigate the relaxor behaviour at different temperatures. Observed ferroelectric behaviour at higher temperature $\left(>T_{m}\right)$ in the present case supported interacting cluster model proposed by Vugmeister and Rabitz. ${ }^{10}$ Two materials systems are considered in the present study which include (a) Pb-based $\mathrm{Pb}\left(\mathrm{Mg}_{1 / 3} \mathrm{Nb}_{2 / 3}\right) \mathrm{O}_{3}-\mathrm{PbTiO}_{3}$ (PMN-PT) type relaxor and (b) Ca-doped $\mathrm{BaTiO}_{3}$ type relaxor. The results obtained on these compositions are discussed individually.

\section{Experimental}

Several fabrication processes have been adapted to develop good quality ferroelectric thin films. These include both chemical and physical vapour deposition such as metal organic chemical vapour deposition (MOCVD), ${ }^{11}$ pulsed excimer laser ablation, ${ }^{12}$ radio frequency sputtering, ${ }^{13}$ sol-gel ${ }^{14}$ etc. In the present work pulsed excimer laser ablation technique (PLD) has been used to deposit relaxor thins. In all cases platinum coated silicon wafers were used as substrates. The details of the processing were described elsewhere. ${ }^{12}$ The structural analysis was done on both types of films by means of X-ray diffraction technique. Microstructure analysis by scanning electron microscope revealed out that the films consist of dense grain structure. Gold electrodes of various diameters $0.4-0.6 \mu \mathrm{m}$ were deposited on the thin films using an evaporation technique to do electrical characterization in metal-insulator-metal configuration. Polarisations vs applied electric field hysteresis studies were done with a RT-66A standardised ferroelectric test system. Dielectric and impedance analysis were carried out, with an oscillation level of $50 \mathrm{mV}$, using an automated HP impedance (4294A) impedance meter in a frequency range of $0 \cdot 1-1 \mathrm{MHz}$ over a temperature range of $-80-450^{\circ} \mathrm{C}$. Dielectric modulus characteristics 
were also evaluated simultaneously on these samples, in order to understand the microstructure dependence on the dielectric and electrical properties in these thin films.

\section{Results and discussions}

\section{$3.1 \mathrm{~Pb}\left(\mathrm{Mg}_{1 / 3} \mathrm{Nb}_{2 / 3}\right) \mathrm{O}_{3}-\mathrm{PbTiO}_{3}$ based system}

3.1a Dielectric phase transition: The results obtained for the dielectric constant $(\varepsilon)$ and dissipation factor $\left(\tan \delta\right.$ ) of the $0.7 \mathrm{PMN}-0.3 \mathrm{PT}$ films, grown at $600^{\circ} \mathrm{C}$ as a function of temperature at different frequencies are shown in figure 1a. The room temperature dielectric constant $(\varepsilon)$ exhibited by the films grown on $\mathrm{LSCO} / \mathrm{Pt} / \mathrm{Ti} / \mathrm{SiO}_{2} / \mathrm{Si}$ substrates is 3600 at the frequency of $1 \mathrm{kHz}$. The dispersion in the temperature of dielectric maxima with frequency as observed in the above figure confirms the relaxor in $0.7 \mathrm{PMN}-0.3 \mathrm{PT}$ films. Dielectric loss peak $\left(\varepsilon^{\prime \prime}\right)$ shown in figure $1 \mathrm{~b}$ also exhibits strong frequency dispersion, indicating that a dielectric property was the intrinsic response from the bulk of the films. This particular nature is a characteristic property of relaxor ferroelectrics, indicates that the system freezes to a glassy state below a certain temperature, ${ }^{15}$ where they take more time to respond to external small $a c$ signal.

3.1b Vogel-Fulcher behaviour: A spin glass state is generally viewed as a system of interacting superparamagnetic clusters ${ }^{16}$ and not as a system of clusters with independent localized behaviour. The universal characteristics of a spin glass, and in general any glassy behaviour, is the existence of a static freezing temperature. In structural glasses the freezing temperature, commonly known as the glass transition temperature, is determined by the onset of measurable visco-elastic behaviour on observable time scales. ${ }^{17}$ In spin glasses a static freezing temperature can be determined from the frequency dependence of the permittivity, ${ }^{18}$ which approaches a constant value at lower frequencies. The freezing of the magnetic moment in spin glasses is believed to arise due to competing interactions, both ferromagnetic and antiferromagnetic, between neighbouring clusters preventing the establishment of conventional long-range ordering. ${ }^{19}$ Instead, these systems become fru-
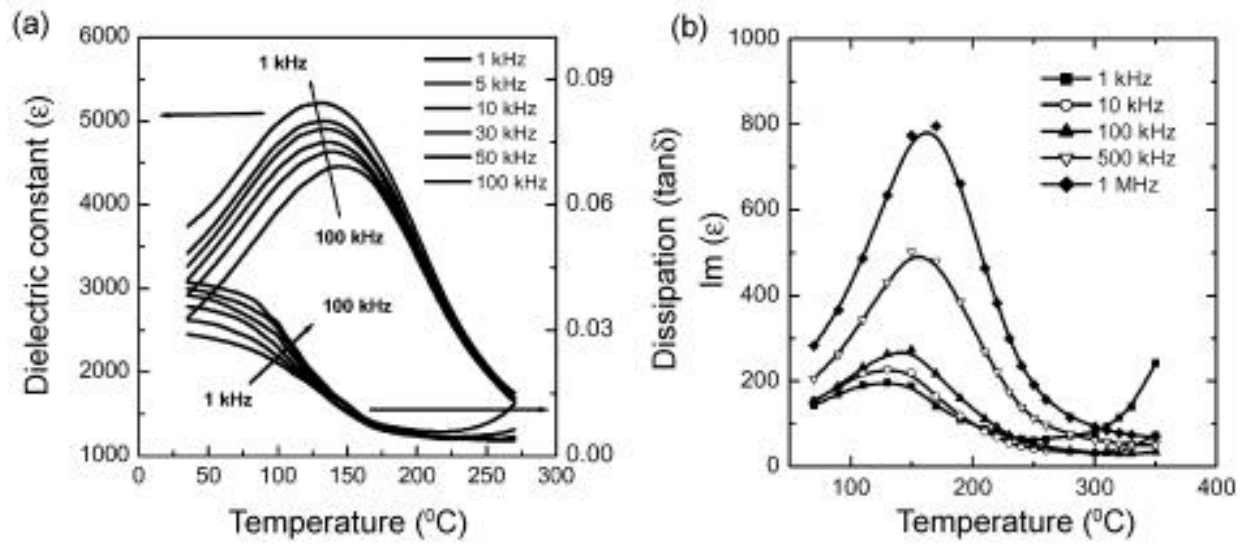

Figure 1. Dielectric phase transition of real and imaginary part of dielectric constant of 07PMN-0.3PT thin films. 
strated by establishing only local ordering, ferromagnetic or antiferromagnetic, between clusters. A dipolar glass is believed to exist due to a competition between ferroelectric and antiferroelectric (e.g. $\mathrm{Rb}_{1-x}\left(\mathrm{NH}_{4}\right)_{x} \mathrm{H}_{2} \mathrm{PO}_{4}$ ). All known glassy systems to date posses two key elements: (1) randomness due an underlying disorder usually with clustering, and (2) frustration leading to freezing.

The magnetic relaxation in spin-glass systems $^{20}$ can be described by a relationship attributed to Vogel and Fulcher given by,

$$
\omega=f_{0} \exp \left[-E_{a} / k\left(T_{\max }-T_{f}\right)\right]
$$

where $\omega$ is the measurement frequency, $T_{f}$ is the static freezing temperature, $E_{a}$ the activation energy, $f_{0}$ the Debye frequency, and $T_{\max }$ the temperature of the permittivity maximum. The activation energy is generally believed to be the product of anisotropy energy, $K_{\text {anis }}$, and the cluster volume, $V$, as originally described by Neel. The analysis provided by our group ${ }^{12}$ for PMN-PT (70/30) composition the obtained frequency dispersion near the transition gave an activation energy of $0.038 \mathrm{eV}$, a pre-exponential factor of $3.5 \times 10^{13} \mathrm{~Hz}$, and a static freezing temperature of $401 \mathrm{~K}$. The curve fitting to the data is shown as the solid line in figure 2.

The close agreement of the data with Vogel-Fulcher relationship, suggests that the relaxor behaviour in the present PMN system is analogous to a spin glass with polarisation fluctuations above a static freezing temperature.

The Vogel-Fulcher relationship may be interpreted as a normal Debye relaxation with temperature-dependent activation energy. The activation energy increases as the temperature decreases becoming undefined as the freezing temperature is approached. A more realistic interpretation of this relationship in reference to relaxors, is that $E_{a}$ represents the activation energy for polarisation fluctuations of an isolated cluster with temperature dependence arising from the development of short-range order between neighbouring clusters with $k T_{f}$ being a measure of the interaction energy. It is certainly possible that the ferroelectric clusters interact via dipole and dipole-induced dipole

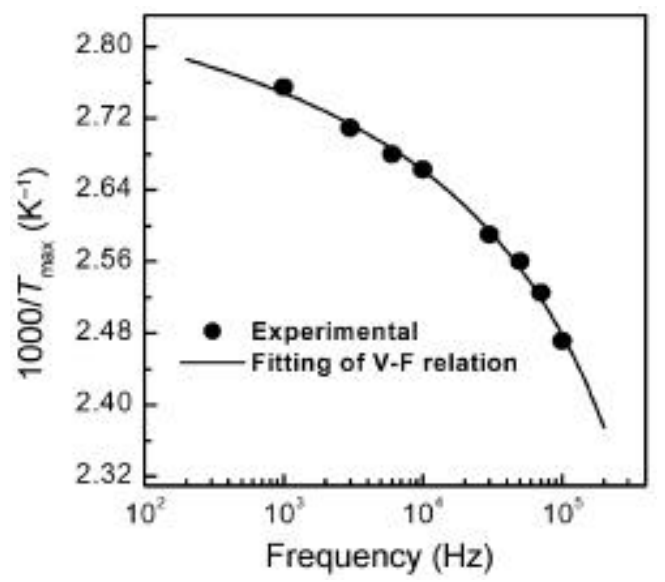

Figure 2. The temperature of permittivity maximum $\left(T_{m}\right)$ as a function of frequency. The points correspond to the experimental data while the solid line is fitting of VogelFulcher relationship. 
exchanges. The possibility also exists that the clusters could interact elastically through local rhombohedral distortions, implying that the clusters may freeze into an orientational glassy state and are superparaelastic above the freezing temperature.

The superparaelectric model, the Vogel-Fulcher dipole glass and dipolar dielectric model are all based on the assumption that the mechanism of the response is related to the thermally activated reorientation of the local spontaneous polarisation in small (about 100 A diametre) polar regions distributed in non-polar matrix.

As has been perceived till now it appears that disorder in the B-site to be a necessary condition for the observed dielectric relaxation. However there are still questions concerning the fundamental physical origin of the dispersion behaviour. Furthermore two different models with very different implications have described the structure of the chemically ordered domains. In the first model, the ordering is $1: 1 \mathrm{Mg}: \mathrm{Nb}$, leading to the presence of $0.5 e^{-}$charge per ordered unit cell. This would imply that the ordered domain contains a space charge and would therefore have its growth limited by the electrostatic interactions. The second model describes the ordered structure as being possessing an intrinsic B-site disorder even in 'fully' ordered crystals. According to this model, there should be no limitation on the ordered domain growth and one should be able to obtain full order in the crystal. Davies ${ }^{21}$ has shown that the ordered structure can be made to spread in the entire crystal in $\mathrm{Pb}\left(\mathrm{Mg}_{1 / 3} \mathrm{Ta}_{2 / 3}\right) \mathrm{O}_{3}$ (PMT), a similar relaxor ferroelectric to PMN. Another important observation found by Davies et $a l^{22}$ shows that even for fully ordered PMT, relaxor behaviour is still present, probably maintained by the intrinsic disorder of the B-site as described above. In the case of PST when the structure is fully ordered, the relaxor behaviour disappears, as there is no more B-site disorder.

\subsection{Field-induced phase transformations}

3.2a Impact of amplitude of ac drive: Large frequency dispersion of dielectric properties at low temperature has been considered as the characteristic property of relaxor ferroelectrics. Such behaviour of relaxor ferroelectrics is due to the presence of number of polar clusters each of which has a net dipole moment. The dipole moment of each cluster has a characteristic response time determined by their local field configuration. The dipole moments have different stable energy minima, which are separated from others by an energy barrier with height of the order of the thermal energy $(25 \mathrm{meV})$ or a little higher. In presence of any $a c$ or $d c$ field, the observed dielectric properties would correspond to the cumulative response of all polar clusters. The number of polar clusters responding to the external field, depends on several factors; such as (a) ambient temperature, (b) amplitude of the $a c$ signal and (c) level of $d c$ bias. As the temperature increases, the height of the energy barrier between the different minima is reduced, which in turn enhances the number of responding polar entities. Similar phenomenon can be visualized by increasing the amplitude of $a c$ drive. Tagantsev et $a l^{9}$ called this individual polar entity a 'relaxator'. These relaxators aredivided into two categories.

Relaxators, which respond to external signal are called the 'fast relaxators' and those which do not, are called 'slow relaxators'. The ratio of these two types keeps on changing with the measurement conditions. With increasing the external field, the number of the fast relaxators could be enhanced so as with temperature.

Figures $3 \mathrm{a}$ and $\mathrm{b}$ represent the dielectric response of $0.7 \mathrm{PMN}-0.3 \mathrm{PT}$ thin films (real and imaginary part of dielectric constant) as a function of $a c$ signal amplitudes measured at different frequencies. The temperature during the dielectric measurement was $40^{\circ} \mathrm{C}$. 
The dielectric constant $\left(\varepsilon^{\prime}\right)$ increases nonlinearly with increasing the oscillation amplitude up to a certain level and finally tends to saturate at higher magnitude of $a c$ drives. Similar phenomenon is also observed in case of imaginary part of dielectric constant. Figure 2 shows the dielectric constant measured at the frequency of $10 \mathrm{kHz}$ as a function of $a c$ drive amplitude at different temperatures. It is interesting to note that dielectric nonlinearity is observed only up to the certain temperature $\left(<T_{m}\right)$. At higher temperatures $\left(>T_{m}\right)$ the dielectric constant is almost independent of oscillation amplitude.

Figures $4 \mathrm{a}-\mathrm{c}$ demonstrate the effect of $a c$ drive amplitudes on dielectric phase transition of PMN-PT (70/30) thin films. With increasing $a c$ drive amplitude, the maximum dielectric transition temperature shifted towards the lower value. Figure 5 displays the temperature-dependence of dielectric constant measured at various ac drives amplitudes at a frequency of $100 \mathrm{kHz}$.

Interestingly, with the increase of $a c$ drive amplitude, the temperature of maximum dielectric constant $\left(T_{m}\right)$ shifts to the lower value. Another important feature to be noticed here is that at higher $a c$ drive fields; the dielectric constant continues to show the frequency dispersive characteristics at the temperature well above the transition temperature. The dispersion at lower temperatures $\left(\sim 40^{\circ} \mathrm{C}\right)$ (shown in figure 5) is also observed to enhance with increasing $a c$ amplitudes. Large frequency dispersion in the dielectric permittivity could be attributed to the contribution similar to the domain wall movement, becomes more prominent at higher $a c$ drive. In relaxor ferroelectrics the regions separating different polar clusters are considered as the domain walls. ${ }^{23}$ As the amplitude of the $a c$ drive increases the contribution from the domain walls dominate over the intrinsic response of the nano polar regions. Moreover, the large electric field could modify the entire random field effect, which is being considered as one of the popularly supported origins of relaxor nature.

3.2b Effect of $d c$ bias: The $d c$ field dependence of the dielectric response, both real and imaginary parts of dielectric constant is depicted in figures $6 a$ and $b$, respectively. It is evident from figures that electric field initially increases the dielectric constant up to a certain value and then reduces. The large frequency dispersion in relaxor ferroelectrics at low field is due to the wide distribution of the height of the potential barriers among which the flipping of the dipoles occurs. The fluctuation results in the changes of the
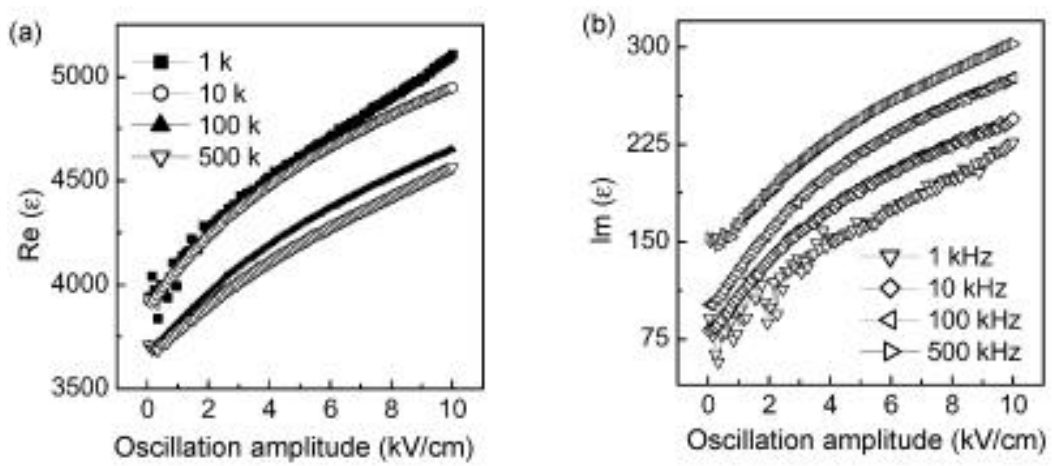

Figure 3. (a) Real and (b) imaginary part of dielectric constant $(\varepsilon)$ as a function of $a c$ drive amplitude at different frequencies. 



Figure 4. Dielectric phase transition of $0 \cdot 7 \mathrm{PMN}-0.3 \mathrm{PT}$ thin films for different oscillation amplitudes: (a) 1 , (b) 5 , and (c) $10 \mathrm{kV} / \mathrm{cm}$.

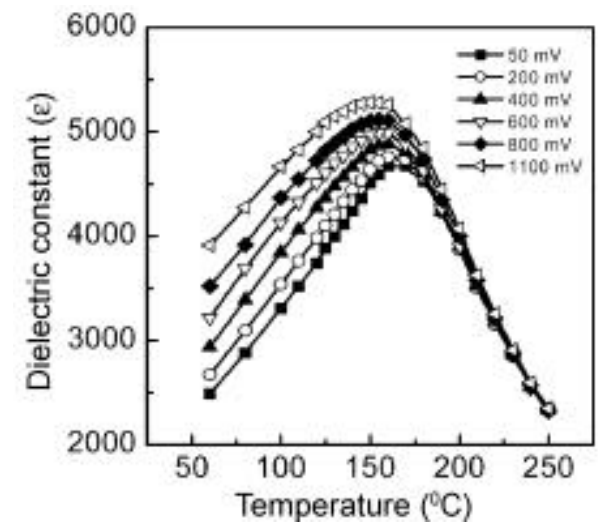

Figure 5. Temperature dependence of dielectric constant measured at $100 \mathrm{kHz}$ for different oscillation amplitudes.

corresponding relaxation times. In presence of weak $d c$ electric bias, the potential barriers between different energy minima are lowered, which intern enhances the flipping 
probabilities of the polarizing entities. ${ }^{24}$ At the same time, the dipoles parallel to the external field are less likely to change their directions. Accumulative response of these two kinds of dipoles all together increases the value of dielectric constant at lower bias.

But the situation becomes reverse when the magnitude of the bias exceeds certain limit. After a certain external bias, the potential barriers for the dipoles parallel to the external field become deeper; therefore, the flipping probability of these dipoles decreases gradually. The number of dipoles will be reducing gradually with increasing bias level since they are frozen along the bias direction. Since the frozen dipoles do not contribute to the orientation dielectric response, the value of the dielectric permittivity is reduced significantly. One more interesting feature that may be observed from the figure that the bias field corresponds to the peak increases with increasing the measuring frequency. Figures $7 \mathrm{a}-\mathrm{d}$ represent the dependence of $d c$ bias on dielectric phase transition of PMN-PT thin films.

The frequency dispersion as evidence from the figures is reduced with increasing bias level while $T_{m}$ follows the reverse trend. This behaviour of the relaxor ferroelectric could be interpreted in terms of locking the moments of polar clusters due to application of external $d c$ bias. As the bias increases, the moments parallel to the electric field increase and finally reach the saturation. Therefore, very few dipoles with narrow spectrum of relaxation times are left to response to the small signal oscillatory electric field. This in turn reduces the magnitude of dielectric constant along with the reduction of frequency dispersion. Since the external $d c$ bias increases potential barriers of the different energy minima, additional thermal energy is required to overcome these potential barriers, resulting in an increase in the phase transition temperature.

Figure 7d shows an interesting feature. A normal ferroelectric-paraelectric phase transition is observed in the temperature-dependence of dielectric constant measured in presence of $30 \mathrm{kV} / \mathrm{cm}$ external bias. This result in turn leads to the inference that the relaxor ferroelectric can be converted to normal ferroelectric with the application of external $d c$ bias. However, there exists symmetry breaking at the nanometre scale, ${ }^{25}$ leading to the formation of polar nanodomains that exist well above the peak in the
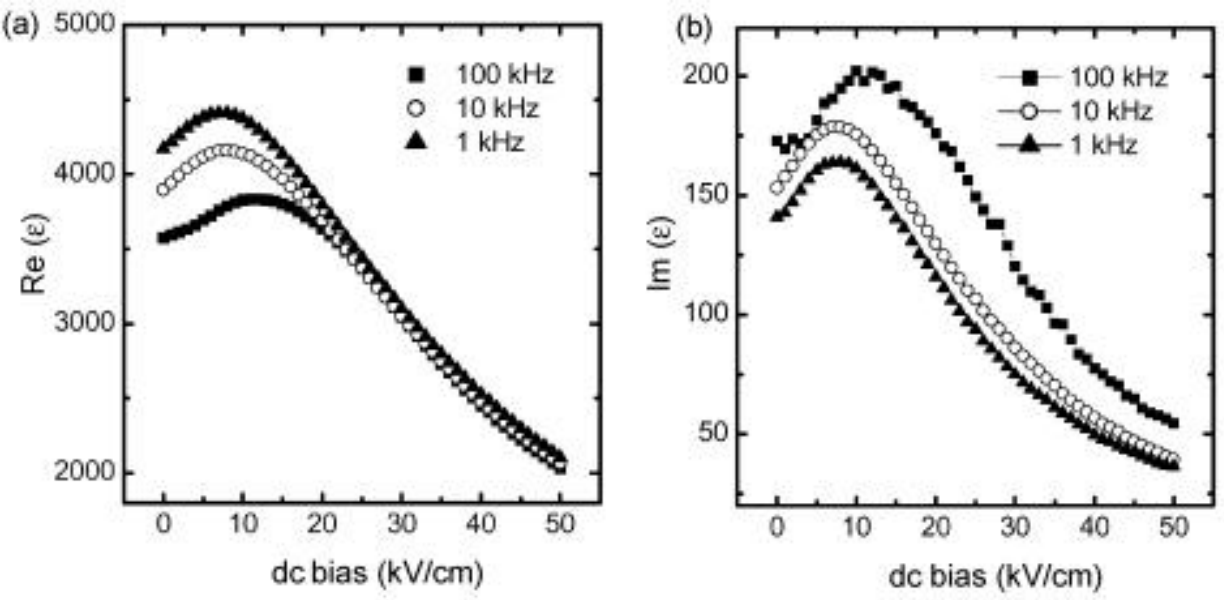

Figure 6. (a) Real and (b) imaginary part of dielectric constant measured at different frequencies as a function of $d c$ bias. 

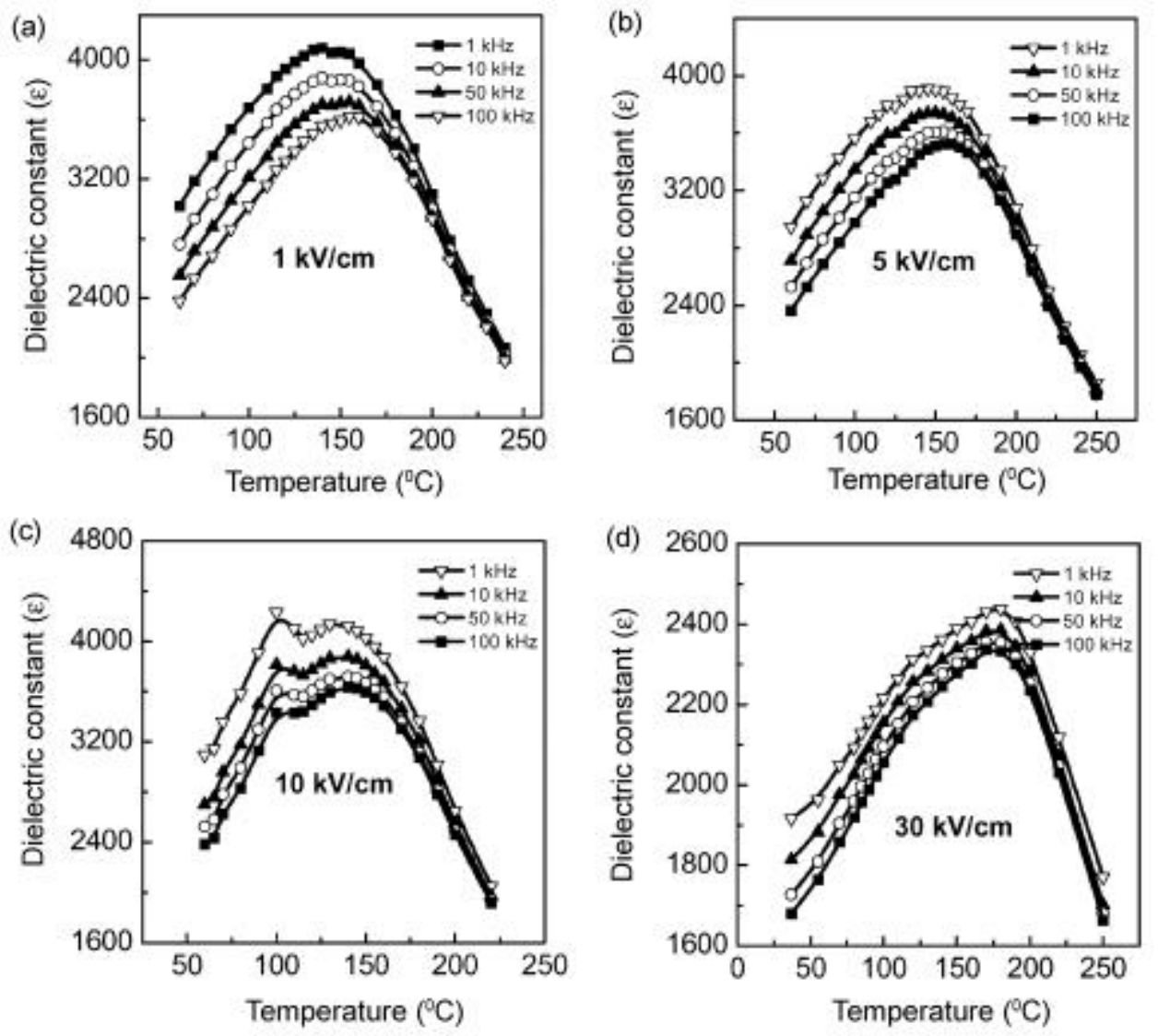

Figure 7. Dielectric phase transition of 0.7PMN-0.3PT thin films after application of different $d c$ bias. (a) 1 , (b) 5, (c) 10 , and (d) $30 \mathrm{kV} / \mathrm{cm}$ respectively.

susceptibility and strongly affects the relaxor properties. As the external bias level exceeds a certain value, where fluctuations of the moments of the polar clusters have condensed, the electric field may override the chemical inhomogeneity, which normally prevents the long range polar ordering. Therefore, $T_{\max }$ could approach the value of homogeneous state due to the destruction of glassy character of relaxors by a large applied electric field. From the close investigation of the figures $7 b$ and $c$, it is interesting to note that in addition to highly dispersive maxima in the temperature range of 130 to $180^{\circ} \mathrm{C}$, additional maxima appeared at approximately $100^{\circ} \mathrm{C}$. The amplitude of these maxima was found to be frequency dependent, whereas the temperature appears to be frequency independent.

\subsection{Ca-doped $\mathrm{BaTiO}_{3}$ system}

Recently, there has been a huge demand for the non lead-based relaxor materials, because of the environmental concerns. Hence the barium-based non lead relaxor materials have been of recent interest as the relaxors have a wide range of applications in the 
microelectromechanical systems (MEMS), piezo electric actuators, multilayer capacitors, and pyroelectric detectors. ${ }^{26,27}$ It is well known fact that the $\mathrm{BaTiO}_{3}$ and its solid solutions have higher piezoelectric and ferroelectric properties. The barium-based relaxor has an advantage in the microwave communications as these have higher tunability and the temperature coefficient of resonance can be made nearly to zero. A series of impurity doped $\mathrm{BaTiO}_{3}$ systems such as $\mathrm{Sn}, \mathrm{Hf}, \mathrm{Ce}$ and $\mathrm{Y}$ have also shown a ferroelectric to relaxor behaviour at higher doping concentrations. ${ }^{28} \mathrm{Ba}\left(\mathrm{Zr}_{x} \mathrm{Ti}_{1-x}\right) \mathrm{O}_{3}$ ceramics and thin films have received considerable amount of attention recently because of its high strain level and high piezoelectric effect. At the lower concentration of $x \sim 0 \cdot 15$, it results in a pinched phase transition and for the $x>0.25$ results in a relaxor diffuse type behaviour. BZT is found to possess composition the dependent property changes. The relaxor behaviour in case of BZT has been attributed due to heterogeneity of the nanoscale composition. ${ }^{29}$ We have observed similar behaviour to relaxor behaviour in a ferroelectric in the case of the Ca-substituting $\mathrm{BaTiO}_{3}$ thin films, which highly dependent on the possibility of the $\mathrm{Ca}$ ions occupying the $\mathrm{Ti}$ site. ${ }^{30}$ The Ca-substituting $\mathrm{BaTiO}_{3}$ crystals and ceramics finds prominent use in the field of optoelectronic devices. ${ }^{31}$ Doping of the $\mathrm{Ca}$ in the $\mathrm{BaTiO}_{3}$ (denoted as BCT) ceramics showed remarkable improvement in electromechanical behaviour, increase in the temperature range of the stability of the tetragonal phase and inhibition in the formation of the unwanted hexagonal phase of $\mathrm{BaTiO}_{3}$.

3.2a Dielectric dispersive behaviour: The dielectric phase transition temperatures of the $\mathrm{BaTiO}_{3}$ thin films are shown in the figure $8 \mathrm{a} . \mathrm{BaTiO}_{3}$ thin film assumes the perovskite form and is observed to be tetragonal, $(c / a>1)$ thereby elongating along an edge below $396 \mathrm{~K}$, which signals the onset of ferroelectricity and a replacement of the centrosymmetric cubic structure by a polar one.

On further cooling, another polymorphic transition occurs at $296 \mathrm{~K}$, with the elongation of the cube along the face diagonal rather than an edge, resulting in a orthorhombic
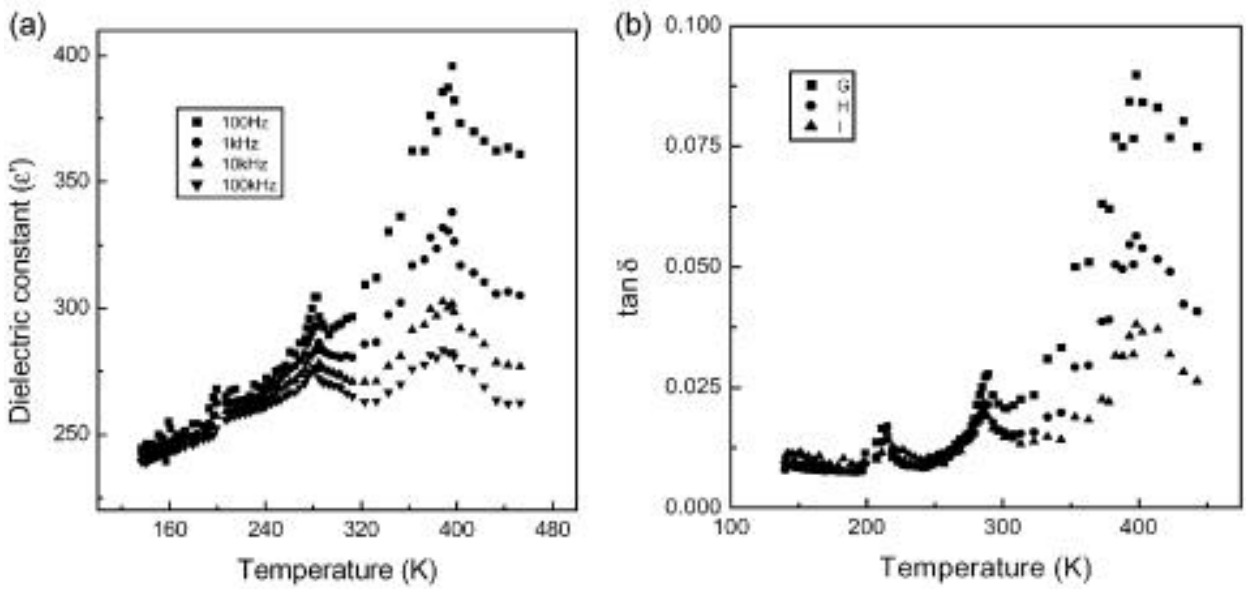

Figure 8. (a) Phase transition temperature of laser-ablated barium titanate thin films. (b) $\tan \delta$ vs temperature of the laser ablated $\mathrm{BaTiO}_{3}$ thin films displaying three phase transitions. 
structure. At even lower temperatures $199 \mathrm{~K}$, a third displacive transformation occurs, with the elongation along a body diagonal and resulting in a rhombohedral structure. The $\tan \delta$ dependence on the temperature is also observed and shows a three distinct phase transition temperatures as shown in the figure $8 b$.

The dielectric phase transition temperature of the $\left(\mathrm{Ba}_{0.9} \mathrm{Ca}_{0.1}\right) \mathrm{TiO}_{3}$ targets was at $380 \mathrm{~K}$ and the $\tan \delta$ dependence on the temperature also follows the similar trend as the dielectric constant versus temperature but peaks out at slightly lower temperature and is in accordance with the Kramers-Kronig model. ${ }^{28}$ They still exhibit a sharper phase transition for even at higher $\mathrm{Ca}$ substituting $\mathrm{Ba}$ in $\mathrm{BaTiO}_{3}$ targets.

The dielectric constant and the $\tan \delta$ dependence on the temperature of the $\mathrm{BC} 125 \mathrm{mt}$ thin films are shown in the figures $9 \mathrm{a}$ and $\mathrm{b}$. A diffuse phase transition (DPT) behaviour was observed for these thin films while the lower concentration of the $\mathrm{Ca}$ doped $\mathrm{BaTiO}_{3}$ thin films showed sharp ferroelectric phase transition. There was an anomalous decrease in the tetragonal phase transition temperature for BC125mt thin films $\left(T_{c} \sim 268\right.$ at $\left.1 \mathrm{kHz}\right)$. The anomalous decrease in the phase transition temperature can be attributed to several reasons such as, integranular stresses, ferroelectric domain sizes, ${ }^{32}$ interface between the substrate and the thin film, possibility of $\mathrm{Ca}^{2+}$ occupancy in $\mathrm{Ti}^{4+}$ site and compositional inhomogenity. ${ }^{33,34}$ From the scanning electron micrograph it is observed that the grain size are quite larger $(\sim 45$ to $80 \mathrm{~nm})$, thereby ignoring the grain size effects and only other possibility is the occupancy of the $\mathrm{Ca}^{2+}$ in $\mathrm{Ti}^{4+}$ site. There are earlier reports on the decrease in the $T_{C}$ to $293 \mathrm{~K}$ for $\mathrm{Ba}_{0.95} \mathrm{Ti}_{1-x} \mathrm{Ca}_{0.05+x} \mathrm{O}_{3+x}(x=0.05)$ ceramics and $320 \mathrm{~K}$ for the $\mathrm{BaTi}_{1-y} \mathrm{CayO}_{3-y}(y=0.05)$ ceramics. ${ }^{35}$

3.2b Composition induced phase transformation: The higher concentration of $\mathrm{Ca}$ (Ca> 10 at.\%) entering the $\mathrm{Ba}$ site, we observed that it still holds the normal ferroelectric behaviour. At the lower concentrations of the $\mathrm{Ca}$ entering the $\mathrm{Ti}$ site it was observed to exhibit a sharper phase transition, while when the higher $\mathrm{Ca}$ enters $(\mathrm{Ca}>10$ at.\%) the $\mathrm{Ti}$ site, it results in more complexity in the compositional modification, thereby leading to a signature of relaxor like behaviour in the BCT thin films and eventually decreasing the
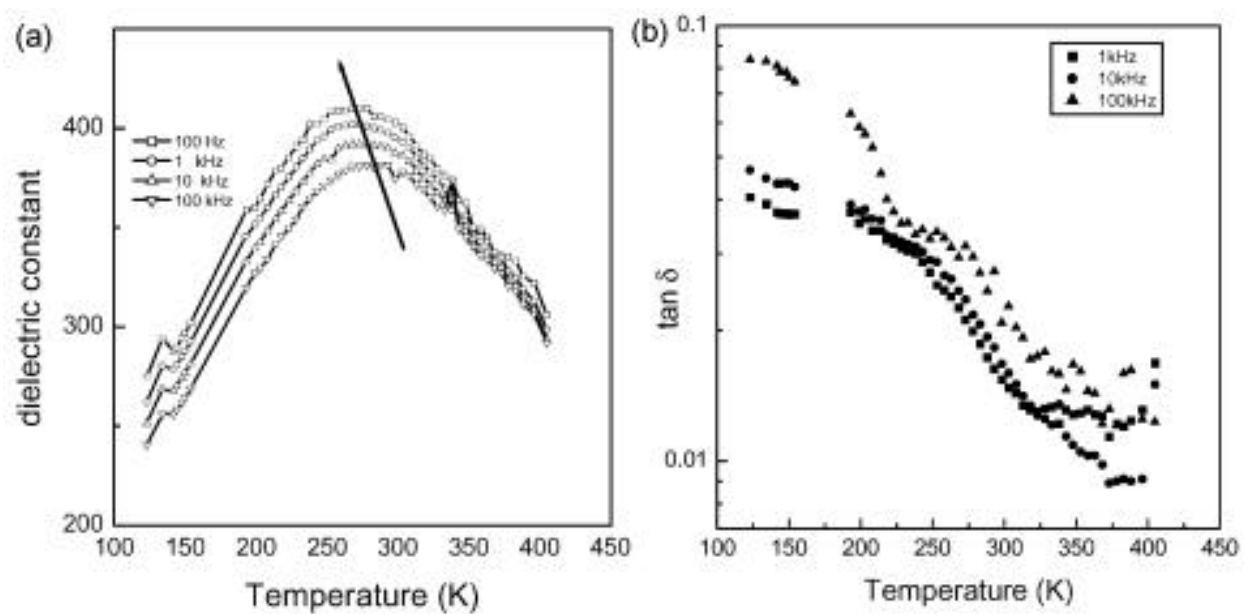

Figure 9. Temperature dependence of (a) dielectric constant and (b) ( $\tan \delta$ ) of BC125mt thin films. 
phase transition temperature. Assuming the distribution of the local transition temperatures of microregions in a DPT material, with microscopic compositional fluctuations and on assuming it to be Gaussian around the mean Curie point $T_{m}$ Kirillov and Isupov ${ }^{36}$ have shown that the

$$
\left(\frac{1}{\varepsilon^{\prime}-\varepsilon_{\infty}}\right)=\left(\frac{1}{\varepsilon_{m}^{\prime}}\right) \exp \left(\left(T-T_{m}^{\prime}\right) 2 \boldsymbol{\delta}^{2}\right),
$$

with $2 \delta$ is the width of the Gaussian and determines the extent of diffuseness of phase transition. If $\varepsilon^{\prime} \gg \varepsilon_{\infty}$, then in the series expansion, higher than second order can be neglected yielding $\gamma=2$. The limiting values $\gamma=1$ and $\gamma=2$ reduces the expression (1) to the Curie-Weiss law valid for the case of a normal ferroelectric and to the quadratic dependence valid for an ideal relaxor respectively. The quadratic dependence of $1 / \varepsilon^{\prime}$ on temperature has been claimed to be obeyed by several materials with diffuse phase transition behaviour. Then $\gamma$ value obtained from a least-squares fit of $\ln \left((1 / \varepsilon)-\left(1 / \varepsilon_{m}^{\prime}\right)\right)$ vs $\ln \left(T-T_{m}^{\prime}\right)$ plot is 1.78 for the BC125mt thin films and is shown in the figure 10 . The diffuseness of the phase transition can be described by an empirical parameter, ${ }^{37}$

$$
\Delta T_{\text {diff }}=T_{0 \cdot 9 \varepsilon}(100 \mathrm{~Hz})-T_{\varepsilon m}(100 \mathrm{~Hz}),
$$

i.e. the difference between $T_{0.9}(100 \mathrm{~Hz})$ (the temperature corresponding to $90 \%$ of the maximum dielectric constant $\left(\varepsilon_{m}\right)$ in the high temperature side) and $T_{\varepsilon m}$ (maximum $\varepsilon$ at temperature for $100 \mathrm{~Hz}$ ). The calculated diffuseness $\Delta T_{\mathrm{dif}}$ for the $\mathrm{BC} 125 \mathrm{mt}$ thin films is $70 \mathrm{~K}$.

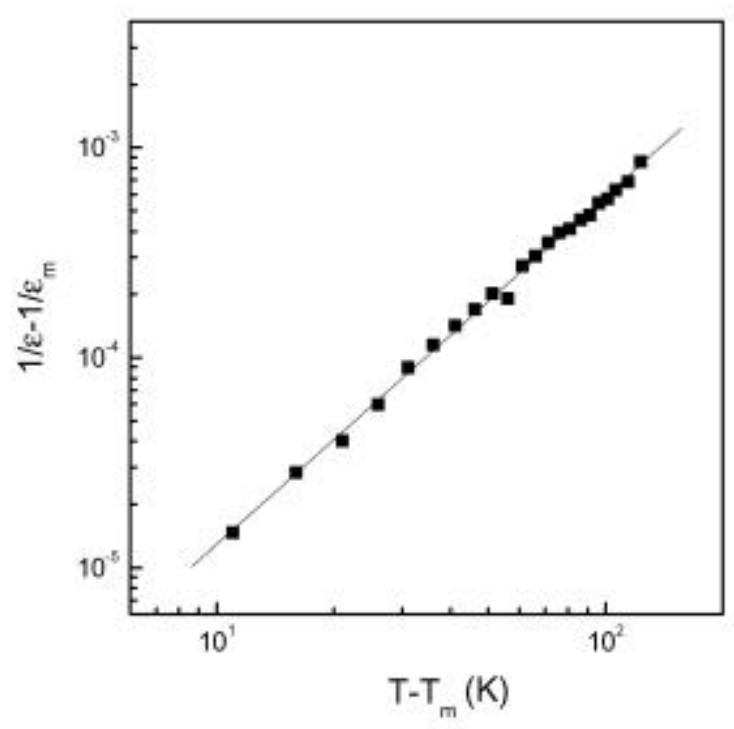

Figure 10. The plot of $\left(1 / \varepsilon-1 / \varepsilon_{m}^{\prime}\right)$ vs $\left(T-T_{m}^{\prime}\right)$ of BC125mt thin films in log-log scale. 
On the other hand, the degree of relaxation behaviour can be described by a parameter.

$$
\Delta T_{\text {relax }}=T_{\varepsilon m}(100 \mathrm{kHz})-T_{\varepsilon m}(100 \mathrm{~Hz}),
$$

with $T_{\varepsilon m}(100 \mathrm{kHz})$ and $T_{\varepsilon m}(100 \mathrm{~Hz})$ denotes the temperature at which maximum $\varepsilon$ was measured at $100 \mathrm{kHz}$ and $100 \mathrm{~Hz}$ respectively. The degree of the relaxation behaviour measured in BCT12C thin films was $10 \mathrm{~K}$. The empirical characterizations with the Curie-Weiss law and the estimated parameters $\left(\Delta T_{\text {relax }}, \Delta T_{\text {diff }}\right.$ and $\left.\gamma\right)$ show that the $\varepsilon$ of the $\mathrm{BC} 125 \mathrm{mt}$ thin films, exhibit a significant diffusiveness of the phase transition and frequency dispersion displaying a relaxor like behaviour in Ca-doped $\mathrm{BaTiO}_{3}$ thin films.

The BC25mt thin films shows a normal ferroelectric behaviour and a normal phase transition temperature as observed in the BCT targets, indicating that the $\mathrm{Ca}$ substitutes in the $\mathrm{Ba}$ site. This shows that the processing - property correlations are important for BCT thin films though we do not intend to go in to detail about them in this paper. The simple substitution of $\mathrm{Ca}^{2+}$ and $\mathrm{Ba}^{2+}$ does not generate a charged defect.

In case, if $\mathrm{Ti}^{4+}$ substitutes $\mathrm{Ca}^{2+}$ in the $\mathrm{BaTiO}_{3}$ lattice, it will act as acceptors to trap the unlocalised electrons. It gives rise to a doubly charged acceptor centre $\mathrm{Ca}_{\mathrm{Ti}}^{\prime \prime}$, which compensates the oxygen vacancies formed, resulting in the formation of high resistivity dielectrics as below.

$$
\mathrm{BaO}+\mathrm{CaO} \rightarrow \mathrm{Ba}_{\mathrm{Ba}}+\mathrm{Ca}_{\mathrm{Ti}}^{\prime \prime}+2 \mathrm{O}_{\mathrm{o}}+\mathrm{V}_{0}^{\prime \prime}
$$

Several reports exist on the possibility of the $\mathrm{Ca}$ substituting the $\mathrm{Ti}$ site in the $\mathrm{BaTiO}_{3}$ ceramics, based on the neutron diffraction, Raman spectra and conductivity measurements. ${ }^{38,39}$ It is suggested that, whenever $(\mathrm{Ba}+\mathrm{Ca}) / \mathrm{Ti}>1$, the $\mathrm{Ca}^{2+}$ would be forced to occupy the $\mathrm{Ti}^{4+}$ site. In the case of the $\mathrm{BC} 125 \mathrm{mt}$, the diffuse phase transition were broad and appeared to be a relaxor type, which could been possibility of either the co-existence of the two phases in thermal equilibrium or the occupancy of $\mathrm{Ca}^{2+}$ entering $\mathrm{Ti}^{4+}$ ions in the $\mathrm{BaTiO}_{3}$ thin films, leading to a compositional and structural disorder on the symmetry breaking. This is brought about by differences in valence ( $2+$ vs $4+$ ) and ionic radii (0.99 vs $0.69 \AA$ ) which is believed to be responsible for the relaxor behaviour. The diffuse phase transition may arise due to the higher composition of the $\mathrm{Ca}$ content entering the $\mathrm{B}$ site disrupting the dipoles, creating a higher degree of disorder, thereby inducing relaxor behaviour. Such behaviour introduces structural randomness, lattice defects, lattice strains and altered forces. The symmetry breaking occurs at the nanometer scale leading to the formation of polar nanodomains which exist well above $T_{\max }$ and increases in size on cooling, but never become large enough to precipitate a long-range-ordered ferroelectric state at $T_{\max }$.

\section{Conclusions}

Even though extensive research work has been carried out in the field of relaxor materials both in theoretical directions as well as in providing experimental evidences for the development of theories, yet a complete universal picture of the origin of the relaxor behaviour is yet to be established. The origin of relaxor behaviour in PMN, PMNT, BCT has been found to be completely different form the other prototype tungsten bronze structure based relaxors. 
Ferroelectric relaxor in thin film form gives rise to another open direction for research. The basic problem arises in the establishment of ferroelectric phase in these thin films. Variety of techniques has been used to suppress the formation of pyrochlore phase formation and to enhance the perovskite phase formation. In thin films the relaxor behaviour has been observed to be greatly influenced by the small grain size leading to a suppression of the relaxor behaviour and broadening of the transition width. Further, very few evidences of the theoretical models have been found in thin films. A future challenge lies in providing satisfying answers to the above problems.

\section{References}

1. Cross L E 1987 Ferroelectrics 76241

2. Smolenskii G A 1965 Sov. Phys. Solid State 61676

3. Viehland D, Jang S, Cross L E and Wittig M 1990 J. Appl. Phys. 682916

4. Bokov V A and Myl'nikova I E 1961 Sov. Phys. Solid State 3613

5. Westphal V, Kleeman W and Glinchuk M D 1992 Phys. Rev. Lett. 68847

6. Viehland D, Li J F, Jang S J, Cross L E and Wittig M 1992 Phys. Rev. B46 8013

7. Qian H and Bursill L A 1996 Int. J. Mod. Phys. B10 2007

8. Chen I-W, Li P and Wang Y 1996 J. Phys. Chem. Solids 571525

9. Glazounov A E and Tagantsev A K 1998 Appl. Phys. Lett. 75856

10. Vugmeister and Rabitz 2000 Phys. Rev. B 6114448

11. Shimizu M and Shiosaki T 1996 Mater. Res. Soc. Symp. Proc. 401129

12. Laha A, Saha S and Krupanidhi S B 2003 Thin Solid Films 424274

13. Jiang M C, Wu T B and Wu J M 1995 Jpn. J. Appl. Phys. 343153

14. Kighelman Z et al 1998 Appl. Phys. Lett. 732281

15. Viehland D, Jang S, Cross L E and Wuttig M 1991 Philos. Mag. B64 335

16. Bitoh T, Obba K, Takamatsu M, Shirane T and Chikazawa S 1996 J. Magn. Magn. Mater.154 59

17. Duffrene L, Gy R, Brlet H and Piques R 1997 J. Non-Cryst. Solids 215208

18. Labarta A, Batlle X, Martinez B and Obradors X 1992 Phys. Rev. B46 8994

19. Toystolytkin A I, Belous N A and Lezhnenko I V 1994 J. Magn. Magn. Mater. 130293

20. Goya G F, Rechenberg H R and Sagredo V 2001 J. Magn. Magn. Mater. 2261298

21. Akbas M A and Davies P K 1997 J. Am. Ceram. Soc. 802933

22. Davies P K, Tong J and Negas T 1997 J. Am. Ceram. Soc. 801727

23. Glazounov A E, Tagantsev A K and Bell A J 1996 Phys. Rev. B53 11281

24. Zhang Y, Gui H, Li L and Gui Z 1997 Jpn. J. Appl. Phys. 36 L1325

25. Burns G and Dacol F H 1983 Phys. Rev. B28 2527

26. Kingon A I, Streiffer, Basceri C and Summerfelt S R 1996 Mater. Res. Soc. Bull. 21(7) 46

27. Auciello O, Scott J F and Ramesh R 1998 Phys. Today 5122

28. Jaffe B, Cook W R and Jaffe H 1971 Piezoelectric ceramics (R.A.N. Publishers)

29. Yu Z, Ang C, Guo R and Bhalla A 2002 J. Appl. Phys. 922655

30. Victor P, Ranjith R, Sarkar A, Vinayak R, Saha S and Krupanidhi S B 2002 Proc. Mater. Res. Soc. U 12.5

31. Veenhuis et al 2000 Appl. Phys. B70 797

32. Shaw T M, Trolier McKinstry S and McIntyre P C 2000 Annu. Rev. Mater. Sci. 30263

33. Zhang, Han Y H, Lal M and Smyth D M 1987 J. Am. Ceram. Soc. 70100

34. Han Y H, Appleby J B and Smyth D M 1987 J. Am. Ceram. Soc. 7096

35. Park J G, Oh T S and Kim Y H 1992 J. Mater. Sci. 275713

36. Kirilov V V and Isupov V A 1973 Ferroelectrics 53

37. Smolenskii G A and Agranovskya A I 1958 Sov. Phys. Tech. Phys. 31380

38. Krishna P S R, Dhananjai Pandey, Tiwari V S, Chakravarthy R and Dasannacharya B A 1993 Appl. Phys. Lett. 62231

39. Chang M C and Chan Yu S C 2000 J. Mater. Sci. Lett. 191323 